\title{
Uniform hypergraphs with the first two smallest spectral radii
}

\author{
Jianbin Zhang ${ }^{a *}$ Jianping $\mathrm{Li}^{b \dagger}$, Haiyan Guo ${ }^{a \ddagger}$ \\ ${ }^{a}$ School of Mathematical Sciences, South China Normal University, \\ Guangzhou 510631, P. R. China \\ ${ }^{b}$ Faculty of Applied Mathematics, Guangdong University of Technology, \\ Guangzhou 510090, P. R. China
}

\begin{abstract}
The spectral radius of a uniform hypergraph $G$ is the the maximum modulus of the eigenvalues of the adjacency tensor of $G$. For $k \geq 2$, among connected $k$-uniform hypergraphs with $m \geq 1$ edges, we show that the $k$-uniform loose path with $m$ edges is the unique one with minimum spectral radius, and we also determine the unique ones with second minimum spectral radius when $m \geq 2$.
\end{abstract}

AMS classification: 05C50, 05C65

KEY WORDS: spectral radius, adjacency tensor, uniform hypergraph edge.

\section{Introduction}

Let $G$ be a hypergraph with vertex set $V(G)$ and edge set $E(G)$, where $E(G)$ is a set whose elements are subsets of $V(G)$. For an integer $k \geq 2$, if each edge of $G$ contains exactly $k$ distinct vertices, then $G$ is a $k$-uniform hypergraph. Two vertices $u$ and $v$ are adjacent if $u$ and $v$ are contained in some edge. An $e$ is incident with vertex $v$ if $v \in e$. An alternating sequence of vertices and edges is called a path if all vertices and edges are distinct, and a cycle if the first and last vertices are the same, the other vertices and all edges are distinct. If there exists a path between any two vertices of $G$, then $G$

\footnotetext{
${ }^{*}$ Corresponding author. E-mail:zhangjb@scnu.edu.cn

${ }^{\dagger}$ E-mail:lijp06@gdut.edu.cn

†E-mail:ghaiyan@163.com
} 
is connected. A hypertree is a connected acyclic hypergraph. A vertex of degree one is called a pendant vertex.

For a $k$-uniform hypergraph $G$ with vertex set $V(G)=\{1, \ldots, n\}$, its adjacency tensor is the tensor $\mathcal{A}(G)=\left(a_{i_{1} \ldots i_{k}}\right)$ of order $k$ and dimension $n$ with $a_{i_{1} \ldots i_{k}}=\frac{1}{(k-1) !}$ if $\left\{i_{1} \ldots i_{k}\right\} \in E(G), 0$ otherwise, where $i_{j} \in\{1, \ldots, n\}$ and $j \in\{1, \ldots, k\}$.

For some complex $\lambda$, if there exists a nonzero vector $x=\left(x_{1}, \ldots, x_{n}\right)^{T}$ such that $\mathcal{A}(G) x=\lambda x^{k-1}$, then $\lambda$ is called an eigenvalue of $G$ and $x$ is called the eigenvector of $G$ corresponding to $\lambda$, where $\mathcal{A}(G) x$ is a $n$-dimensional vector whose $i$-th component is

$$
(\mathcal{A}(G) x)_{i}=\sum_{i_{2}, \ldots, i_{k}=1}^{n} a_{i i_{2} \ldots i_{k}} x_{i_{2}} \cdots x_{i_{k}}
$$

and $x^{k-1}=\left(x_{1}^{k-1}, \ldots, x_{n}^{k-1}\right)^{T}$, Moreover, if $\lambda$ and $x$ are both real, then we call $\lambda$ an $H$-eigenvalue of $G$. Note that $x^{T}(\mathcal{A}(G) x)=k \sum_{e \in E(G)} \prod_{v \in e} x_{v}$.

The spectral radius of a $k$-uniform hypergraph $G$, denoted by $\rho(G)$, is defined as the maximum modulus of eigenvalues of $\mathcal{A}(G)$. Since $\mathcal{A}(G)$ is symmetric and thus $\rho(G)$ is the largest $H$-eigenvalue of $\mathcal{A}(G)$, see [13]. It is proved in [4, 12] that for a connected $k$-uniform hypergraph $G, \mathcal{A}(G)$ has a unique positive eigenvector $x$ with $\sum_{v \in V(G)} x_{v}^{k}=1$ corresponding to $\rho(G)$, which is called the principal eigenvector of $G$.

The problem to determine the hypergraphs in some given classes of hypergraphs with maximum spectral radius received much attention. Li et al. [5] determined the uniform hypertree with maximum spectral radius. Yuan et al. 17] extended to this to determine the first eight uniform hypertrees with maximum spectral radius. Fan et al. [3] determined the hypergraphs with maximum spectral radius among uniform hypergraphs with few edges. Xiao et al. [15] determined the hypertree with maximum spectral radius among uniform hypertrees with a given degree sequence. See [] for more work on hypergraphs with maximum spectral radius in someclasses of uniform hypergraphs. However, there is much less work on the problem to determine the hypergraphs in some given classes of hypergraphs with minimum spectral radius. Li et al. [5] determined the unique one with minimum spectral radius among $k$-uniform power hypertrees with fixed number of edges. Here a $k$-uniform power hypertree is a $k$-uniform hypertree in which every edge contains at least $k-2$ pendant vertices. Lu and Man [7] classified all connected $k$-uniform hypergraphs with spectral radius at most $\sqrt[k]{4}$. There seems no more result in this line. In this note, for $k \geq 2$, we show that the $k$-uniform loose path with $m$ edges is the unique one with minimum spectral radius among connected $k$-uniform hypergraphs with $m \geq 1$ edges, and we also determine the unique ones with second minimum spectral radius among connected $k$-uniform hypergraphs with $m \geq 2$ edges. To obtain our main 
result, we use the nice result (Lemma 60 from [7] and propose a hypergraph transformation that decreases the spectral radius (in Lemma 4).

\section{Preminaries}

Let $r \geq 1, G$ be a hypergraph with $u \in V(G)$ and $e_{1}, \ldots, e_{r} \in E(G)$. Suppose that $v_{i} \in e_{i}$ and $u \notin e_{i}$ for $i=1,2, \ldots, r$. Let $e_{i}^{\prime}=\left(e_{i} \backslash\left\{v_{i}\right\}\right) \cup\{u\}$ for $i=$ $1, \ldots, r$. Suppose that $e_{i}^{\prime} \notin E(G)$ for $i=1, \ldots, r$. Let $G^{\prime}$ be the hypergraph obtained from $G$ by deleting $e_{1}, e_{2}, \ldots, e_{r}$ and adding $e_{1}^{\prime}, e_{2}^{\prime}, \ldots, e_{r}^{\prime}$. Then we say that $G^{\prime}$ is obtained from $G$ by moving $\left(e_{1}, e_{2}, \ldots, e_{r}\right)$ from $\left(v_{1}, v_{2}, \ldots, v_{r}\right)$ to $u$.

Lemma 1 [5] Let $r \geq 1, G$ be a hypergraph with $u \in V(G)$ and $e_{1}, \ldots, e_{r} \in$ $E(G)$. If $G^{\prime}$ is obtained from $G$ by moving $\left(e_{1}, e_{2}, \ldots, e_{r}\right)$ from $\left(v_{1}, v_{2}, \ldots, v_{r}\right)$ to $u$, and $x_{u} \geq \max _{1 \leq i \leq r} x_{v_{i}}$, then $\rho\left(G^{\prime}\right)>\rho(G)$.

Lemma 2 [15] Let $G$ be a connected $k$-uniform hypergraph, and $e=U_{1} \cup U_{2}$, $f=V_{1} \cup V_{2}$ be two edges of $G$, where $e \cap f=\emptyset$, and $1 \leq\left|U_{1}\right|=\left|V_{1}\right|<k$. Let $e^{\prime}=U_{1} \cup V_{2}$ and $f^{\prime}=V_{1} \cup U_{2}$. Suppose that $e^{\prime}, f^{\prime} \notin E(G)$. Let $G^{\prime}$ be the hypergraph obtained from $G$ by deleting edges $e$ and $f$ and adding edges $e^{\prime}$ and $f^{\prime}$. Let $x$ be the principle eigenvector of $G$. If $x_{U_{1}} \geq x_{V_{1}}, x_{U_{2}} \leq x_{V_{2}}$, and one is strict, then $\rho(G)<\rho\left(G^{\prime}\right)$.

A path $\left(u_{0}, e_{1}, u_{1}, \ldots, e_{p}, u_{p}\right)$ in a $k$-unoform hypergraph $G$ is called a pendant path at $u_{0}$ if $d\left(u_{0}\right) \geq 2, d\left(u_{i}\right)=2$ for $i=1,2, \ldots, p-1, d\left(u_{p}\right)=1$ and $d(u)=1$ for any $u \in e_{i} \backslash\left\{u_{i-1}, u_{i}\right\}$ with $i=1,2, \ldots, p$. If $p=1$, then it is a pendant edge at $u_{0}$.

For a $k$-uniform hypergraph $G$ with a pendant path $P$ at $u$, we say that $G$ is obtained from $H$ by attaching a pendant path $P$ at $u$, where $H=$ $G[V(G) \backslash(V(P) \backslash\{u\})]$. We write $G=H(u, p)$ if the length of $P$ is $p$. Let $H(u, 0)=H$.

For a $k$-unifoorm hypergraph $G$ with $u \in V(G)$, and $p \geq q \geq 0$, let $G_{u}(p, q)=\left(G_{u}(p)\right)_{u}(q)$.

Lemma 3 [16] Let $u$ be a vertex of a connected $k$-uniform hypergraph $G$ with $|E(G)| \geq 1$. If $p \geq q \geq 1$, then $\rho\left(G_{u}(p, q)\right)>\rho\left(G_{u}(p+1, q-1)\right)$.

Let $G$ be a connected $k$-uniform hypergraph with $u, v \in V(G)$, and $p \geq$ $q \geq 0$, let $G_{u, v}(p, q)=\left(G_{u}(p)\right)_{v}(q)$.

Lemma 4 Let $G$ be a $k$-uniform hypergraph with $k \geq 3$. Let e be a pendant edge of $G$, and $u$ and $v$ be two pendant vertices in e. If $p \geq q \geq 1$, then $\rho\left(G_{u, v}(p, q)\right)>\rho\left(G_{u, v}(p+1, q-1)\right)$. 
Proof. Suppose that $P=\left(u_{1}, e_{1}, u_{2}, e_{2}, \ldots, u_{p+1}, e_{p+1}, u_{p+2}\right)$ and $Q=\left(v_{1}, f_{1}\right.$, $\left.v_{2}, f_{2}, \ldots, v_{q-1}, f_{q-1}, v_{q}\right)$ are two pendant paths of $G_{u, v}(p+1, q-1)$ at $u$ of length $p+1$ and at $v$ of length $q-1$, respectively, where $u=u_{1}$ and $v=v_{1}$. If $q=1$, then let $Q=\left(v_{1}\right)$.

Suppose that $\rho\left(G_{u, v}(p, q)\right) \leq \rho\left(G_{u, v}(p+1, q-1)\right)$. Let $x$ be the principal eigenvector of $G_{u, v}(p+1, q-1)$.

Suppose that $x_{u_{p+1}} \leq x_{v_{q}}$. Let $G^{\prime}$ be the hypergraph obtained from $G_{u, v}(p+1, q-1)$ by moving edge $e_{p+1}$ from $u_{p+1}$ to $v_{q}$. It is obvious that $G^{\prime} \cong$ $G_{u, v}(p, q)$. By Lemma 1, we have $\rho\left(G_{u, v}(p, q)\right)=\rho\left(G^{\prime}\right)>\rho\left(G_{u, v}(p+1, q-1)\right)$, a contradiction. Hence $x_{u_{p+1}}>x_{v_{q}}$.

Suppose that $i \geq 1$ and $x_{u_{p+1-i}}>x_{v_{q-i}}$ for $i \leq q-2$. We want to show that $x_{u_{p-i}}>x_{v_{q-i-1}}$.

Suppose that $x_{u_{p-i}} \leq x_{v_{q-i-1}}$. If $x_{e_{p-i} \backslash\left\{u_{p-i}, u_{p-i+1}\right\}}>x_{f_{q-i-1} \backslash\left\{v_{q-i-1}, v_{q-i}\right\}}$. Let $U_{1}=e_{p-i} \backslash\left\{u_{p-i}\right\}$ and $V_{1}=f_{q-i-1} \backslash\left\{v_{q-i-1}\right\}$. Let $G^{\prime}$ be the hypergraph $G^{\prime}$ obtained from $G_{u, v}(p+1, q-1)$ by deleting edges $e_{p-i}$ and $f_{q-i-1}$ and adding edges $e_{p-i}^{\prime}$ and $f_{q-i-1}^{\prime}$, where $e_{p-i}^{\prime}=U_{1} \cup\left(f_{q-i-1} \backslash V_{1}\right)$ and $f_{q-i-1}^{\prime}=V_{1} \cup\left(e_{p-i} \backslash\right.$ $\left.U_{1}\right)$. Note that $G^{\prime} \cong G_{u, v}(p, q)$. We have by Lemma 2 that $\rho\left(G_{u, v}(p, q)\right)=$ $\rho\left(G^{\prime}\right)>\rho\left(G_{u, v}(p+1, q-1)\right)$, a contradiction. Thus $x_{e_{p-i} \backslash\left\{u_{p-i}, u_{p-i+1}\right\}} \leq$ $x_{f_{q-i-1} \backslash\left\{v_{q-i-1}, v_{q-i}\right\}}$. Now let $U_{1}=\left\{u_{p-i+1}\right\}$ and $V_{1}=\left\{v_{p-i}\right\}$. Let $G^{\prime}$ be the hypergraph obtained from $G_{u, v}(p+1, q-1)$ by deleting edges $e_{p-i}$ and $f_{q-i-1}$ and adding edges $e_{p-i}^{\prime}$ and $f_{q-i-1}^{\prime}$, where $e_{p-i}^{\prime}=V_{1} \cup\left(e_{p-i} \backslash U_{1}\right)$ and $f_{q-i-1}^{\prime}=U_{1} \cup\left(f_{q-i-1} \backslash V_{1}\right)$. Note that $G^{\prime} \cong G_{u, v}(p, q)$. By Lemma 2, we have $\rho\left(G^{\prime}\right)=\rho\left(G_{u, v}(p, q)\right)>\rho\left(G_{u, v}(p+1, q-1)\right)$, also a contradiction. It follows that $x_{u_{p-i}}>x_{v_{q-i-1}}$, as desired.

Let $e=\left\{u_{1}, v_{1}, w_{1}, w_{2}, \ldots, w_{k-2}\right\}$ with $d_{G}\left(w_{1}\right) \geq 2$ and let $e_{p-q+1}=$ $\left\{u_{p-q+1}, u_{p-q+2}, w_{1}^{\prime}, w_{2}^{\prime}, \ldots, w_{k-2}^{\prime}\right\}$. Clearly, $x_{w_{2}}=\cdots=x_{w_{k-2}}$ and $x_{w_{1}^{\prime}}=$ $\cdots=x_{w_{k-3}^{\prime}}$. If $x_{w_{1}} \leq x_{w_{1}^{\prime}}$, then we can obtain a $G^{\prime}$ from $G_{u, v}(p+1, q-1)$ by moving all the edges except $e$ incident with $w_{1}$ from $w_{1}$ to $w_{1}^{\prime}$. By Lemma 1 and the fact that $G^{\prime} \cong G_{u, v}(p, q)$, we have $\rho\left(G_{u, v}(p, q)\right)>\rho\left(G_{u, v}(p+1, q-1)\right)$, a contradiction. Hence $x_{w_{1}}>x_{w_{1}^{\prime}}$.

Suppose that $x_{e \backslash\left\{v_{1}, w_{1}\right\}} \geq x_{e_{p-q+1} \backslash\left\{u_{p-q+2}, w_{1}^{\prime}\right\}}$. Let $U_{1}=e \backslash\left\{v_{1}\right\}$ and $V_{1}=$ $e_{p-q+1} \backslash\left\{u_{p-q+2}\right\}$. Thus we can form a hypergraph $G^{\prime}$ from $G_{u, v}(p+1, q-1)$ by deleting edges $e$ and $e_{p-q+1}$ and adding edges $e^{\prime}$ and $e_{p-q+1}^{\prime}$, where $e^{\prime}=$ $U_{1} \cup\left(e_{p-q+1} \backslash V_{1}\right)$ and $e_{p-q+1}^{\prime}=V_{1} \cup\left(e \backslash U_{1}\right)$. It is obvious that $G^{\prime} \cong G_{u, v}(p, q)$. By Lemma 2, we have $\rho\left(G_{u, v}(p, q)\right)>\rho\left(G_{u, v}(p+1, q-1)\right)$, a contradiction. Thus $x_{e \backslash\left\{v_{1}, w_{1}\right\}}<x_{e_{p-q+1} \backslash\left\{u_{p-q+2}, w_{1}^{\prime}\right\}}$. Now let $U_{1}=\left\{w_{1}\right\}$ and $V_{1}=\left\{w_{1}^{\prime}\right\}$. Let $G^{\prime}$ be the hypergraph obtained from $G_{u, v}(p+1, q-1)$ by deleting edges $e$ and $e_{p-q+1}$ and adding edges $e^{\prime}$ and $e_{p-q+1}^{\prime}$, where $e^{\prime}=V_{1} \cup\left(e \backslash U_{1}\right)$ and $e_{p-q+1}^{\prime}=U_{1} \cup\left(e_{p-q+1} \backslash V_{1}\right)$. Note that $G^{\prime} \cong G_{u, v}(p, q)$. By Lemma 2, we have $\rho\left(G^{\prime}\right)=\rho\left(G_{u, v}(p, q)\right)>\rho\left(G_{u, v}(p+1, q-1)\right)$, also a contradiction. We complete the proof.

A hypergraph $G$ is said to be reducible if every edge contains at least one pendant vertex. For a reducible $k$-uniform hypergraph $G$ with $e \in E(G)$, 
let $v_{e}$ be a pendant vertex in $e$, and let $G^{\prime}$ be the hypergraph with $V\left(G^{\prime}\right)=$ $V(G) \backslash\left\{v_{e}: e \in E(G)\right\}$ and $E\left(G^{\prime}\right)=\left\{e \backslash\left\{v_{e}\right\}: e \in E(G)\right\}$. We say that $G^{\prime}$ is reduced from $G$.

Lemma $5 \sqrt{7]}$ Let $G$ be a reducible k-uniform hypergraph. If $G^{\prime}$ is reduced from $G$, then $\rho^{k}(G)=\rho^{k-1}\left(G^{\prime}\right)$.

For a $k$-uniform hypertree $G$ with $E(G)=\left\{e_{1}, \ldots, e_{m}\right\}$, if $V(G)=$ $\left\{v_{1}, \ldots, v_{n}\right\}$ with $n=(k-1) m+1$, and $e_{i}=\left\{v_{(i-1)(k-1)+1}, \ldots, v_{(i-1)(k-1)+k}\right\}$ for $i=1, \ldots, m$, then we call $G$ a $k$-uniform loose path, denoted by $P_{m}^{(k)}$.

For $k \geq 2$ and $m \geq 3$, let $D_{m}^{(k)}$ be the $k$-uniform hypertree obtained from a $k$-uniform loose path $P_{m-1}^{(k)}=\left(u_{0}, e_{1}, u_{1}, \ldots, e_{m-1}, u_{m-1}\right)$ by attaching a pendant edge at $u_{1}$.

For $k \geq 3$ and $m \geq 3$, let $D_{m}^{\prime(k)}$ be the $k$-uniform hypertree obtained from a $k$-uniform loose path $P_{m-1}^{(k)}=\left(u_{0}, e_{1}, u_{1}, \ldots, e_{m-1}, u_{m-1}\right)$ by attaching a pendant edge at a vertex of degree 1 in $e_{2}$.

Lemma 6 [7] Let $G$ be a $k$-uniform hypergraph with $k \geq 3$.

(i) If $k=3$ and $\rho(G)<\sqrt[k]{4}$, then $G$ is isomorphic to one of the following hypergraphs: $P_{m}^{(3)}$ for $m \geq 1, D_{m}^{(3)}$ for $m \geq 3$, $D_{m}^{\prime(3)}$ for $m \geq 4, B_{m}^{(3)}$ for $m \geq 5, B_{m}^{\prime(3)}$ for $m \geq 6, \bar{B}_{m}^{(3)}$ for $m \geq 7, B D_{m}^{(3)}$ for $m \geq 5$, and thirty-one additional hypergraphs: $E_{1,2,2}^{(3)}, E_{1,2,3}^{(3)}, \bar{E}_{1,2,4}^{(3)}, F_{2,3,3}^{(3)}, F_{2,2, l}^{(3)}($ for $2 \leq l \leq 6), F_{1,3, l}^{(3)}$ $($ for $3 \leq l \leq 13), F_{1,4, l}^{(3)}($ for $4 \leq l \leq 7), F_{1,5,5}^{(3)}$, and $G_{1,1: l: 1,3}^{(3)}($ for $0 \leq l \leq 5)$ (see Figure 1).

(ii) If $k=4, G$ is not reducible, and $\rho(G) \leq \sqrt[k]{4}$, then $G \cong H_{1,1,1, t}$ with $t=1,2,3,4$ (see Figure 2).

(iii) If $k \geq 5$ and $\rho(G) \leq \sqrt[k]{4}$, then $G$ is reducible.

We remark that in Figure 1, $E_{i, j, l}^{(3)}$ consists of three pendant paths of length $i, j$ and $l$ at a common vertex, $F_{i, j, l}^{(3)}$ consists of three pendant paths of length $i, j$ and $l$ at three different vertices of a single edge, and $G_{i, j: l: p, q}^{(3)}$ consists of a 3 -uniform loose path of length $l+2$ with two pendant paths of length $i$ and $j$ at two pendant vertices in the first edge and two pendant paths of length $p$ and $q$ at two pendant vertices in the last edge.

\section{Result}

Now we are ready to show our main result.

Theorem 1 Let $G$ be a connected $k$-uniform hypergraph with $m \geq 4$ edges, where $k \geq 3$. Suppose that $G ¥ P_{m}^{(k)}$. Then $\rho(G) \geq \rho\left(D_{m}^{\prime(k)}\right)$ with equality if and only if $G \cong D_{m}^{\prime(k)}$. 

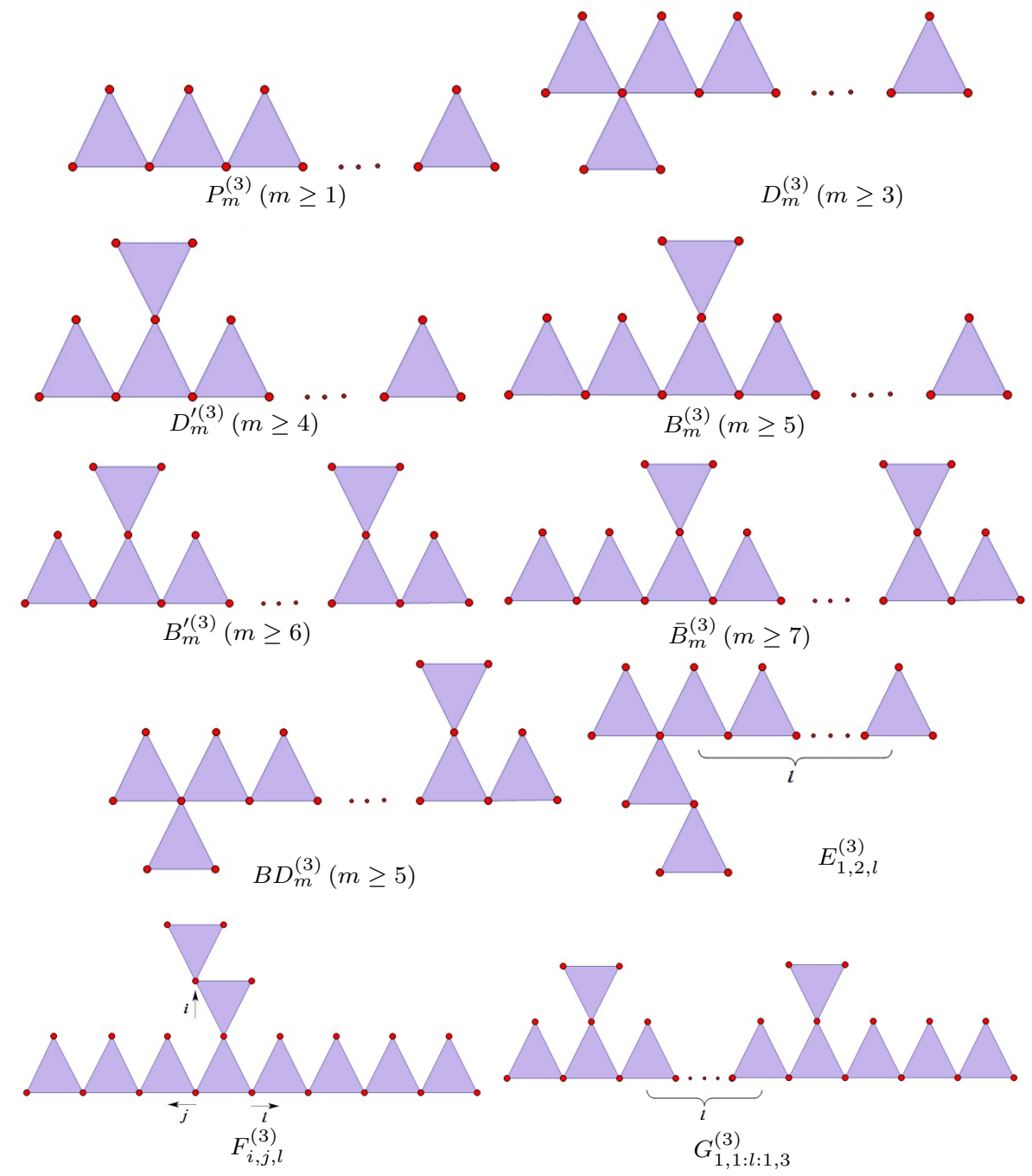

Figure 1: Hypergraphs in Lemma 6(i) 

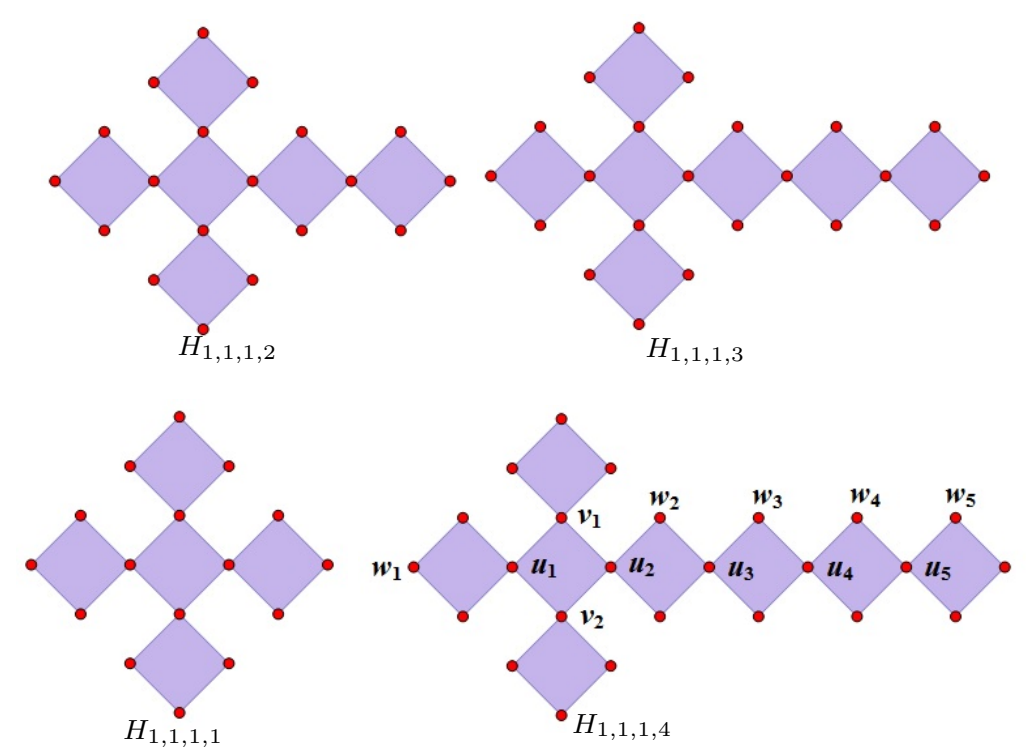

Figure 2: Hypergraphs $H_{1,1,1, i}$ for $i=1,2,3,4$ in Lemma 6(ii).

Proof. By Lemma 6(i) $\rho\left(D_{m}^{\prime(3)}\right)<\sqrt[3]{4}$. Then by Lemma 5, we have $\rho^{k}\left(D_{m}^{\prime(k)}\right)=\rho^{3}\left(D_{m}^{\prime(3)}\right)<4$, and thus $\rho\left(D_{m}^{\prime(k)}\right)<\sqrt[k]{4}$.

Let $G$ be a connected $k$-uniform hypergraph with $m \geq 4$ edges and $G \approx$ $P_{m}^{(k)}$ having minimum spectral radius. We need only to show that $G \cong D_{m}^{(k)}$.

Since $\rho\left(D_{m}^{\prime(k)}\right)<\sqrt[k]{4}$, we have $\rho(G)<\sqrt[k]{4}$.

Case 1. $k=3$.

By Lemma 6(i), $G$ is isomorphic to one of the following hypergraphs: $P_{m}^{(3)}$ for $m \geq 1, D_{m}^{(3)}$ for $m \geq 3, D_{m}^{\prime(3)}$ for $m \geq 4, B_{m}^{(3)}$ for $m \geq 5, B_{m}^{\prime(3)}$ for $m \geq 6$, $\bar{B}_{m}^{(3)}$ for $m \geq 7, B D_{m}^{(3)}$ for $m \geq 5$, and thirty-one additional hypergraphs: $E_{1,2,2}^{(3)}, E_{1,2,3}^{(3)}, E_{1,2,4}^{(3)}, F_{2,3,3}^{(3)}, F_{2,2, l}^{(3)}$ (for $\left.2 \leq l \leq 6\right), F_{1,3, l}^{(3)}($ for $3 \leq l \leq 13), F_{1,4, l}^{(3)}$ (for $4 \leq l \leq 7), F_{1,5,5}^{(3)}$, and $G_{1,1: l: 1,3}^{(3)}($ for $0 \leq l \leq 5)$.

By Lemma 1, we have $\rho\left(D_{m}^{(3)}\right)>\rho\left(D_{m}^{\prime(3)}\right)$.

By Lemma 3 , we have $\rho\left(B D_{m}^{(3)}\right)>\rho\left(D_{m}^{\prime(3)}\right)$, and for $E_{1,2, l}^{(3)}$ with $l=2,3,4$, we have $m=l+3$, and thus $\rho\left(E_{1,2, l}^{(3)}\right)>\rho\left(D_{l+3}^{(3)}\right)>\rho\left(D_{m}^{\prime(3)}\right)$.

By Lemma 4 , we have $\rho\left(B_{m}^{(3)}\right)>\rho\left(D_{m}^{\prime(3)}\right), \rho\left(B_{m}^{\prime(3)}\right)>\rho\left(D_{m}^{\prime(3)}\right)$, and $\rho\left(\bar{B}_{m}^{(3)}\right)>$ $\rho\left(D_{m}^{\prime(3)}\right)$.

For $F_{i, j, l}^{(3)}$, we have $m=i+j+l+1$. By Lemma 4 , we have $\rho\left(F_{i, j, l}^{(3)}\right)>$ $\rho\left(F_{i, 1, m-i-2}^{(3)}\right)>\rho\left(F_{1,1, m-3}^{(3)}\right)=\rho\left(D_{m}^{\prime(3)}\right)$. Thus $\rho\left(F_{2,3,3}^{(3)}\right)>\rho\left(D_{9}^{\prime(3)}\right), \rho\left(F_{2,2, m-5}^{(3)}\right)>$ $\rho\left(D_{m}^{\prime(3)}\right)$ for $(7 \leq m \leq 11), \rho\left(F_{1,3, m-5}^{(3)}\right)>\rho\left(D_{m}^{\prime(3)}\right)$ (for $\left.8 \leq m \leq 18\right)$, $\rho\left(F_{1,4, m-6}^{(3)}\right)>\rho\left(D_{m}^{\prime(3)}\right)($ for $10 \leq m \leq 13)$, and $\rho\left(F_{1,5,5}^{(3)}\right)>\rho\left(D_{12}^{\prime(3)}\right)$.

By Lemma 4, we have $\rho\left(G_{1,1: m-8: 1,3}^{(3)}\right)>\rho\left(F_{1,1, m-3}^{(3)}\right)=\rho\left(D_{m}^{\prime(3)}\right)$ for $8 \leq$ $m \leq 13$.

Therefore, if $G \nsubseteq D_{m}^{\prime(k)}$, then $\rho(G)>\rho\left(D_{m}^{\prime(3)}\right)$. It follows that $G \cong D_{m}^{\prime(3)}$.

Case 2. $k=4$. 
If $G$ is reducible, then by Lemma 5 , for the hypergraph $G_{1}$ reduced from $G$, we have $\rho\left(G_{1}\right)<\sqrt[3]{4}$, and by the proof in Case 1 , we have $G_{1} \cong D_{m}^{\prime(3)}$, implying that $G \cong D_{m}^{\prime(4)}$.

Next suppose that $G$ is not reducible. Then by Lemma 6(ii), $G \cong H_{1,1,1, i}$ with $i=1,2,3,4$. We will show that these are impossible. Since $\rho\left(D_{m}^{(3)}\right)>$ $\rho\left(D_{m}^{\prime(3)}\right)$, by Lemma 3 we have $\rho\left(D_{m}^{(4)}\right)>\rho\left(D_{m}^{\prime(4)}\right)$. Thus it suffices to show that $\rho(G)>\rho\left(D_{m}^{(4)}\right)$.

Suppose that $G \cong H_{1,1,1,4}$. Then $m=8$. From the table of [2] we have $\rho\left(D_{8}^{(2)}\right)=1.962$. By Lemma 3 we have $\rho\left(D_{8}^{(4)}\right)=\left(\rho\left(D_{8}^{(2)}\right)^{\frac{2}{4}}\right.$, implying that $\left(\rho\left(D_{8}^{(4)}\right)\right)^{4}=3.8494$.

Let $x$ be the principal eigenvector of $G$, and let $u_{i}, w_{i}$ for $i=1,2,3,4,5$ and $v_{1}, v_{2}$ be the vertices of $H_{1,1,1,4}$ as labeled in Figure 2). Let $\rho(G)=\rho$ and $x_{u_{i}}=x_{i}$ for $i=1,2,3,4,5$. Then $x_{v_{1}}=x_{v_{2}}=x_{u_{1}}=x_{1}$. We have $\rho x_{w_{1}}^{4}=$ $x_{w_{1}}^{3} x_{1}$, and then $x_{w_{1}}=\frac{x_{1}}{\rho}$. Similarly, we have that $x_{w_{5}}=\frac{x_{5}}{\rho}, x_{w_{i}}=\sqrt{\frac{x_{i} x_{i+1}}{\rho}}$ for $i=2,3,4$. Thus

$$
\begin{aligned}
\rho x_{1}^{4} & =\frac{x_{1}^{3}}{\rho^{3}} x_{1}+x_{1}^{3} x_{2}, \\
\rho x_{2}^{4} & =x_{1}^{3} x_{2}+\frac{x_{2}^{2} x_{3}^{2}}{\rho}, \\
\rho x_{3}^{4} & =\frac{x_{2}^{2} x_{3}^{2}}{\rho}+\frac{x_{3}^{2} x_{4}^{2}}{\rho}, \\
\rho x_{4}^{4} & =\frac{x_{3}^{2} x_{4}^{2}}{\rho}+\frac{x_{4}^{2} x_{5}^{2}}{\rho}, \\
\rho x_{5}^{4} & =\frac{x_{4}^{2} x_{5}^{2}}{\rho}+\frac{x_{5}^{3}}{\rho^{3}} x_{5} .
\end{aligned}
$$

From the first two equations, we have $\left(\rho^{2}-\frac{\rho^{10}}{\left(\rho^{4}-1\right)^{3}}\right) x_{2}^{2}=x_{3}^{2}$, and from the the other three equations, we have $x_{2}^{2}=\rho^{2} x_{3}^{2}-x_{4}^{2}=\left(\rho^{6}-3 \rho^{2}+\frac{1}{\rho^{2}}\right) x_{5}^{2}$ and $x_{3}^{2}=\rho^{2} x_{4}^{2}-x_{5}^{2}=\left(\rho^{4}-2\right) x_{5}^{2}$. Thus

$$
\left(\rho^{4}\right)^{5}-8\left(\rho^{4}\right)^{4}+21\left(\rho^{4}\right)^{3}-23\left(\rho^{4}\right)^{2}+13\left(\rho^{4}\right)-3=0 .
$$

Since $P_{6}^{(4)}$ is a subhypergraph of $G$, we have $\rho^{4}>\rho^{4}\left(P_{6}^{(4)}\right)=\left(\sqrt{2 \cos \frac{\pi}{8}}\right)^{4}=$ $2+\sqrt{2}$.

Let $f(t)=t^{5}-8 t^{4}+21 t^{3}-23 t^{2}+13 t-3$. Note that $f\left(\frac{1}{2}\right)=-\frac{3}{2^{5}}<$ $0, f(2-\sqrt{2})=-7+5 \sqrt{2}>0, f(1)=1>0, f(2+\sqrt{2})=-7-5 \sqrt{2}<$ $0, f(3.9)=-4.94181<0$, and $f(4)=1>0$. Thus $f(t)=0$ has three real roots $t_{1}, t_{2}$ and $t_{3}$ satisfying $\frac{1}{2}<t_{3}<2-\sqrt{2}, 1<t_{2}<2+\sqrt{2}$, and $3.9<t_{1}<4$. Let $t_{4}$ and $t_{5}$ be the remaining two roots of $f(t)=0$. Then $t_{4} t_{5}>0, t_{4}+t_{5}>8-(2-\sqrt{2})-(2+\sqrt{2})-4=0$, and $t_{4}+t_{5}<8-\frac{1}{2}-1-3.9=$ $2.6<2+\sqrt{2}$. Note that $\rho^{4}>2+\sqrt{2}$. So whether $t_{4}$ and $t_{5}$ are real or not, 
they can not be equal to $\rho$. Thus $\rho^{4}=t_{1}>3.9>3.8494=\left(\rho\left(D_{8}^{(4)}\right)^{4}\right.$, i.e., $\rho(G)>\rho\left(D_{8}^{(4)}\right)$, as desired.

In the following, we consider the cases when $G \cong H_{1,1,1, i}$ for $i=1,2,3$. From the table of [2] we have $\rho\left(D_{5}^{(2)}\right)=1.902, \rho\left(D_{6}^{(2)}\right)=1.932$, and $\rho\left(D_{7}^{(2)}\right)=$ 1.950. Then by Lemma 3 we have $\rho\left(D_{5}^{(4)}\right)=1.3791,\left(\rho\left(D_{6}^{(4)}\right)\right)^{4}=3.733$, $\left(\rho\left(D_{7}^{(4)}\right)\right)^{4}=3.8025$. By similar but simpler argument as above, we have $\rho\left(H_{1,1,1,1}\right), \rho\left(H_{1,1,1,2}^{(4)}\right)$, and $\rho\left(H_{1,1,1,3}\right)$ are roots of $\rho^{4}-\rho^{3}-1=0,\left(\rho^{4}\right)^{4}-$ $6\left(\rho^{4}\right)^{3}+10\left(\rho^{4}\right)^{2}-7\left(\rho^{4}\right)+2=0$, and $\left(\rho^{4}\right)^{5}-7\left(\rho^{4}\right)^{4}+15\left(\rho^{4}\right)^{3}-13\left(\rho^{4}\right)^{2}+$ $6\left(\rho^{4}\right)-1=0$, respectively. And we may check that

$$
\begin{array}{ll}
\rho(G)>1.38>1.3791=\rho\left(D_{5}^{(4)}\right) & \text { if } i=1, \\
\rho^{4}(G)>3.8>3.733=\rho^{4}\left(D_{6}^{(4)}\right) & \text { if } i=2, \\
\rho^{4}(G)>3.9>3.8025=\rho^{4}\left(D_{7}^{(4)}\right) & \text { if } i=3,
\end{array}
$$

i.e., $\rho(G)>\rho\left(D_{m}^{(4)}\right)$, as desired.

Case 3. $k \geq 5$.

By Lemma 6(iii) , $G$ is reducible. By Lemma 5, for the hypergraph $G_{1}$ reduced from $G$, we have $\left.\rho\left(G_{1}\right)\right)<\sqrt[k-1]{4}$. Repeating this process by using Lemmas 6(iii) and 5, we have a hypergraph sequence $G_{0}, G_{1}, \ldots, G_{k-4}$ with $G_{0}=G$, where $G_{i+1}$ is reduced from $G_{i}$ for $i=0, \ldots, k-5$. It is easily seen that $G_{k-4}$ is 4-uniform and $\rho^{k}(G)=\rho^{4}\left(G_{k-4}\right)<4$. By the proof of Case 2, $G_{k-4} \cong D_{m}^{(4)}$, and thus $G \cong D_{m}^{(k)}$.

Among connected 2-uniform hypergraphs with $m$ edges, the ones with spectral radius less than 2 are determined in 8 to be the trees $P_{m}^{(2)}, D_{m}^{(2)}$, and three additional trees with $m=5,6,7$, obtained from $D_{m-1}^{(2)}$ by attaching a pendant edge at a pendant vertex that is adjacent to a vertex of degree 3 , and by Lemma 3 , it is easy to see that $P_{m}^{(2)}$ for $m \geq 1$ is the unique one with minimum spectral radius, while $D_{m}^{(2)}$ for $m \geq 3$ is the unique one with second minimum spectral radius.

Let $G$ be a connected $k$-uniform hypergraph with 2 edges, where $k \geq 3$. Let $a$ be the number of common vertices of the two edges. Obviously, $1 \leq a \leq k-1$. By direct calculation, $\rho(G)=2^{\frac{a}{k}}$. Therefore $P_{2}^{(k)}$ and the hypergraph in which two edges share two vertices in common are the unique hypergraphs with minimum and second minimum spectral radii among connected $k$-uniform hypergraphs with exactly 2 edges.

Let $G$ be a connected $k$-uniform hypergraphs with 3 edges, where $k \geq$ 3. If there is a subhypergraph consisting two edges containing at least two vertices in common, then $\rho(G) \geq \sqrt[k]{4}$. If any two edges of $G$ contain at most one common vertex, then $G$ is a cycle of length $3, D_{3}^{(k)}$ or $P_{3}^{(k)}$. If $G$ is a cycle of length 3, then $\rho(G)=\sqrt[k]{4}$. By Lemma 3, $\rho\left(D_{3}^{(k)}\right)>\rho\left(P_{3}^{(k)}\right)$. By Lemmas 5 and 6(i), $\rho^{k}\left(D_{3}^{(k)}\right)=\rho^{3}\left(D_{3}^{(3)}\right)<4$. Therefore $P_{3}^{(k)}$ and $D_{3}^{(k)}$ are 
the unique hypergraphs with minimum and second minimum spectral radii among connected $k$-uniform hypergraphs with exactly 3 edges.

For $m \geq 4$ and $k \geq 3$, by Lemma 4 , we have $\rho\left(D_{m}^{\prime(k)}\right)>\rho\left(P_{m}^{(k)}\right)$.

Combining the above facts and Theorem 1, we have

Theorem 2 Among connected $k$-uniform hypergraphs with $m$ edges, $P_{m}^{(k)}$ for $m \geq 1$ is the unique one with minimum spectral radius, and the hypergraph in which two edges share two vertices in common for $m=2$ and $k \geq 3, D_{3}^{(k)}$

for $k=2$ or $m=3$, and $D_{m}^{\prime(k)}$ for $m \geq 4$ and $k \geq 3$ are the unique ones with second minimum spectral radius.

\section{Acknowledgements}

This work was supported by National Natural Science Foundation of China (No. 11071089 and No. 11701102), Natural Science Foundation of Guangdong Province (No. 2017A030313032 and No. 2017A030310441).

\section{References}

[1] J. Cooper, A. Dutle, Spectra of uniform hypergraph, Linear Algebra Appl. 436 (2012), 3268-3292.

[2] D. Cvetkoić, M. Doob, H. Sachs, Spectra of Graphs-Theory and Applications, Academic Press, New York, 1980.

[3] Y. Fan, Y. Tan, X. Peng, A. Liu, Maximizing spectral radii of uniform hypergraphs with few edges, Discuss. Math. Graph Theory 36 (2016) 845-856.

[4] S. Friedland, S. Gaubert, L. Han, Perron-Frobenius theorem for nonnegative multilinear forms and extensions, Linear Algebra Appl. 438 (2013) 738-749.

[5] H. Li, J. Shao, L. Qi, The extremal spectral radii of $k$-uniform hypertrees, J. Comb. Optim. 32 (2016) 741-764.

[6] P. Keevash, J. Lenz, D. Mubayi, Spectral extremal problems for hypergraphs, SIAM J. Discrete Math. 28 (2014) 1838-1854

[7] L. Lu, S. Man, Connected hypergraphs with small spectral radius, Linear Algebra Appl. 509 (2016) 206-227.

[8] J. H. Smith, Some properties of the spectrum of a graph, in: Combinatorial Structures and their Applications, Gordon and Breach, New York, 1970, pp. 403-406. 
[9] J. Zhang, J. Li, The maximum spectral radius of k-uniform hypergraphs with $r$ pendent vertices, Linear Multilinear Algebra, DOI: 10.1080/03081087.2018.1442811.

[10] V. Nikiforov, Analytic methods for uniform hypergraphs, Linear Algebra Appl. 457 (2014) 455-535.

[11] K. Pearson, Spectral hypergraph theory of the adjacency hypermatrix and matroids, Linear Algebra Appl., 465 (2015), 176-187.

[12] K.J. Pearson, T. Zhang, On spectral hypergraph theory of the adjacency tensor, Graphs Combin. 30 (2014) 1233-1248.

[13] L. Qi, Symmetric nonnegative tensors and copositive tensors, Linear Algebra Appl. 439 (2013) 228-238.

[14] B. Wu, E. Xiao, Y. Hong, The spectral radius of trees on $k$ pendant vertices, Linear Algebra Appl. 395 (2005) 343-349.

[15] P. Xiao, L.Wang, Y. Lu,The maximum spectral radii of uniform hypertree s with given degree sequences, Linear Algebra Appl., 523 (2017), 33-45.

[16] P. Xiao, L. Wang, The maximum spectral radius of uniform hypergraphs with given number of pendant edges, Linear Multilinear Algebra, DOI: 10.1080/03081087.2018.1453471.

[17] X. Yuan, J. Shao, H. Shan, Ordering of some uniform hypertree s with larger spectral radii, Linear Algebra Appl., 495 (2016), 206-222.

[18] J. Zhou, L. Sun, W. Wang, C. Bu, Some spectral properties of uniform hypergraphs, Electron J. Combin. 21 (2014), P4. 24. 